\title{
Thrombospondin-1 restrains neutrophil granule serine protease function and regulates the innate immune response during Klebsiella pneumoniae infection
}

 \\ RK Mallampalli ${ }^{1,2}$, M Sun $^{3}$, RL Silverstein ${ }^{4}$, DB Stolz ${ }^{3}$, SD Shapiro ${ }^{1}$, A Ray ${ }^{1}$, P Ray and JS Lee ${ }^{1,5}$
}

Neutrophil elastase (NE) and cathepsin G (CG) contribute to intracellular microbial killing but, if left unchecked and released extracellularly, promote tissue damage. Conversely, mechanisms that constrain neutrophil serine protease activity protect against tissue damage but may have the untoward effect of disabling the microbial killing arsenal. The host elaborates thrombospondin-1 (TSP-1), a matricellular protein released during inflammation, but its role during neutrophil activation following microbial pathogen challenge remains uncertain. Mice deficient in TSP-1 (thbs $1^{-{ }^{-}}$) showed enhanced lung bacterial clearance, reduced splenic dissemination, and increased survival compared with wildtype (WT) controls during intrapulmonary Klebsiella pneumoniae infection. More effective pathogen containment was associated with reduced burden of inflammation in thbs $1^{-I^{-}}$mouse lungs compared with WTcontrols. Lung NE activity

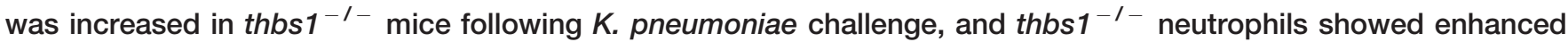
intracellular microbial killing that was abrogated with recombinant TSP-1 administration or WT serum. Thbs $1^{-1-}$ neutrophils exhibited enhanced NE and CG enzymatic activity, and a peptide corresponding to amino-acid residues 793-801 within the type-III repeat domain of TSP-1 bridled neutrophil proteolytic function and microbial killing in vitro. Thus, TSP-1 restrains proteolytic action during neutrophilic inflammation elicited by $K$. pneumoniae, providing a mechanism that may regulate the microbial killing arsenal.

\section{INTRODUCTION}

Thrombospondin-1 (TSP-1) is a matricellular protein that exists as a trimer of identical $\sim 150-180 \mathrm{kDa}$ subunits tethered together by disulfide bonds. ${ }^{1,2}$ A major source of TSP-1 is platelet $\alpha$-granules, with TSP- 1 being released upon platelet activation. ${ }^{3-5}$ TSP-1 also exists in plasma at low concentrations under basal conditions, and is made by numerous cells including myeloid-derived cells such as neutrophils. ${ }^{6,7}$ Given its adhesive nature, TSP-1 can bind to the surface of platelets, extracellular matrix, and a number of other cells including fibroblasts, smooth muscle cells, endothelium, neutrophils, and macrophages, providing a multiplicity of potential cell-cell and cell-matrix interactions. ${ }^{2}$ Thus, it is not surprising that TSP-1 has been implicated in a number of important biological functions such as embryogenesis, wound repair, tumor growth and metastasis, angiogenesis, hemostasis, and inflammation. ${ }^{2,5,8-11}$

Others have previously shown that platelet proteins such as TSP-1 are found in the airspace of patients with acute respiratory distress syndrome and TSP-1 concentrations correlate with composite injury scores that quantify the degree of lung injury. ${ }^{12}$ The original description of mice deficient in TSP-1 (thbs $\left.1^{-/-}\right)$showed spontaneous development of non-infectious pneumonia and the predisposition to develop inflammation due to impaired homeostasis. ${ }^{13}$ We recently

\footnotetext{
${ }^{1}$ Division of Pulmonary, Allergy, and Critical Care Medicine, Department of Medicine, University of Pittsburgh, Pittsburgh, Pennsylvania, USA. ${ }^{2}$ The Medical Specialty Service Line, Veterans Affairs Pittsburgh Healthcare System, Pittsburgh, Pennsylvania, USA. ${ }^{3}$ Department of Cell Biology and Center for Biologic Imaging, University of Pittsburgh, Pittsburgh, Pennsylvania, USA. ${ }^{4}$ Department of Medicine, Medical College of Wisconsin and Blood Research Institute, Blood Center of Wisconsin, Milwaukee, Wisconsin, USA and ${ }^{5}$ Vascular Medicine Institute, Department of Medicine, University of Pittsburgh, Pittsburgh, Pennsylvania, USA. Correspondence: JS Lee (leejs3@upmc.edu)
} 
demonstrated that, although thbs $1^{-/-}$mice do not spontaneously develop non-infectious pneumonia, these mice exhibit a defect in their ability to resolve from injurious stimuli to the lungs. ${ }^{14}$ We have also shown that interleukin (IL)-10 is essential for recovery of lung inflammation during the late phase of bacterial infection. ${ }^{14,15}$ In an experimental model of lung injury, we further show that the bridging function of TSP-1 is required for optimal triggering of macrophage IL-10 production following contact recognition of apoptotic neutrophils that is necessary for effective resolution of inflammation. ${ }^{14,15}$ It remains unclear, however, what the role of TSP-1 is, if any, during bacterial pneumonia, an important cause of morbidity and mortality worldwide and a well-known risk factor for acute respiratory distress syndrome. ${ }^{16}$

Neutrophils are innate immune effector cells of microbial killing and are critical for pulmonary host defense against pathogen. Two major modes of intracellular killing are operant in neutrophils: (1) oxygen-dependent mechanism involving the recruitment and activation of the NADPH oxidase complex, the generation of oxygen free radicals and superoxide, resulting in myeloperoxidase-mediated halogenation to form hypochlorous acid; (2) the release of cytosolic granule contents within the phagosome, comprised of neutrophil serine proteases, the activation of these proteases within the phagosome, ${ }^{17-19}$ and the contribution of antimicrobial proteins. ${ }^{20}$ Neutrophil elastase (NE), a key granule serine protease, degrades outer membrane protein A on the surface of Gram-negative bacteria $^{21}$ and is an important contributor to the oxygenindependent arm of microbial killing. Although effective neutrophil microbial killing is required to thwart collateral tissue damage and organ injury induced by microbial-host interactions, the host also requires mechanisms to curtail its microbial killing arsenal to prevent subsequent collateral tissue damage and organ injury. Indeed, unbridled neutrophil protease activity is associated with lung injury or acute respiratory distress syndrome in humans. ${ }^{22}$ Thus, a fine balance is required for effective neutrophil microbial-killing activity on one hand and the curtailment of an over-vigorous host inflammatory response on the other.

TSP-1 is a competitive inhibitor of serine proteases such as plasmin, preventing the cleavage of fibrinogen in vitro with stoichiometric predictions demonstrating $1 \mathrm{~mol}$ of TSP-1 interacting with $1 \mathrm{~mol}$ of plasmin. ${ }^{23}$ This prompted further studies showing that TSP-1 binds and competitively inhibits the enzymatic activity of purified NE and cathepsin G (CG) in vitro. ${ }^{3,4}$ These findings suggest a regulatory role for TSP-1 during inflammation, but how TSP-1 modulates neutrophil function during the innate immune response to bacterial pathogens is unclear.

\section{RESULTS \\ Increased bacterial clearance from the lungs, reduced splenic dissemination, and enhanced survival following intratracheal instillation of Klebsiella pneumoniae in mice deficient in TSP-1}

To investigate the role of TSP-1 in pulmonary host defense, thbs $1^{-1-}$ mice were inoculated with the bacterial pathogen $K$. pneumoniae at $4 \times 10^{3}$ colony-forming units (CFUs). At 48 and $72 \mathrm{~h}$ following intratracheal inoculation, thbs $1^{-1-}$ mice showed reduced bacterial burden in the lungs compared with wild-type (WT) mice, as measured by CFU/lung (Figure 1a). Consistent with the findings in the lungs, thbs $1^{-1-}$ mice showed reduced splenic dissemination compared with WT mice (Figure 1b). Thus, pulmonary host defense is enhanced during bacterial pneumonia in the absence of TSP- 1 that is associated with reduced systemic dissemination. We next determined whether the enhanced bacterial clearance in thbs $1^{-1-}$ mice confers a survival advantage during bacterial pneumonia with $K$. pneumoniae. At an inoculum of $1.2 \times 10^{4} \mathrm{CFU}$, lethal dose $\left(\mathrm{LD}_{50}\right)$ was achieved at $72 \mathrm{~h}$ in WT mice (Figure 1c). Kaplan-Meier curve followed by the log-rank test showed enhanced survival in thbs $1^{-/-}$mice compared with WT mice $(P=0.02, n=20$ per group). The median survival for thbs $1^{-/-}$mice was $312 \mathrm{~h}$ (13 days), compared with $72 \mathrm{~h}$ (3 days) for WT mice. A kinetics study examining responses of WT and thbs $1^{-1-}$ mice $24,48,72$, and $96 \mathrm{~h}$ following intratracheal inoculation $\left(1.8 \times 10^{3} \mathrm{CFU}\right)$ indicated that, by 72 and $96 \mathrm{~h}, t h b s 1^{-1-}$ mice were clearing bacteria from the lungs faster than WT mice (Supplementary Figure 1 online). Consistent with our prior findings, ${ }^{24}$ thbs $1^{-/}$mice show deficiency in IL-10, which is required for optimal resolution of lung inflammation during the late
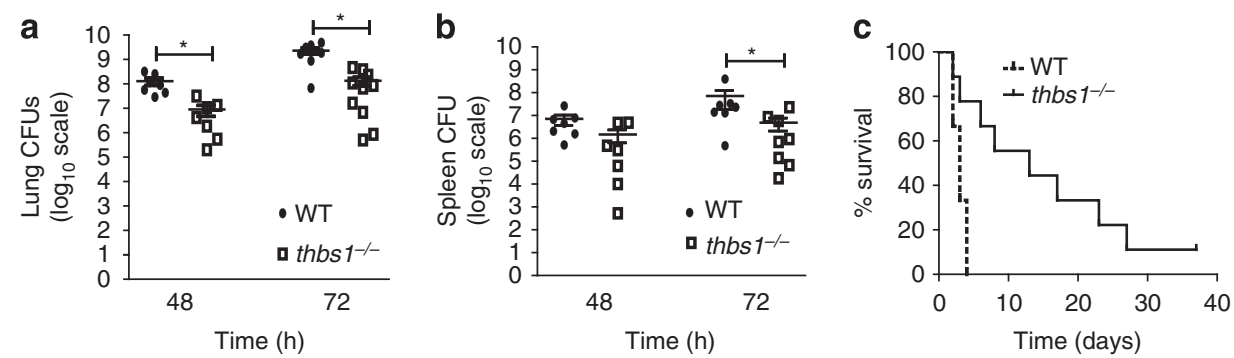

Figure 1 thbs $1^{-/-}$mice show enhanced lung bacterial clearance, reduced splenic dissemination, and enhanced survival following intratracheal K. pneumoniae inoculation. Colony-forming units (CFU) obtained from (a) lung tissue homogenate and (b) splenic homogenate cultures of WT and thbs $1^{-/}-$ mice 48 and $72 \mathrm{~h}$ after intratracheal (i.t.) inoculation with $K$. pneumoniae, $n=7-10$ mice per group. Kruskal-Wallis test with Dunn's multiple comparisons, ${ }^{\star} P<0.05$. (c) A Kaplan-Meier survival curve of WT and thbs $1^{-1-}$ mice following i.t. infection with K. pneumoniae $(n=20$ mice per genotype, $P=0.02)$. The median survival for WT mice was $72 \mathrm{~h}$ ( 3 days) and $312 \mathrm{~h}$ (13 days) for thbs $1^{-/-}$mice. WT, wild type. 
phase after infection. ${ }^{15}$ Taken together, TSP-1 deficiency improves early lung bacterial clearance, reduces systemic dissemination, and confers increased survival during bacterial pneumonia induced by K. pneumoniae. However, thbs $1^{-1-}$ mice may later succumb to sub-optimal resolution from injury due to inability to trigger full IL-10 responses in the lungs.

\section{thbs $1^{-1-}$ mice show enhanced lung NE activity and reduced parenchymal inflammation following intratracheal K. pneumoniae}

TSP- 1 can bind and induce the motility and chemotaxis of neutrophils in vitro, ${ }^{25-28}$ and we have previously reported that thbs $1^{-1-}$ mice show slightly higher leukocyte counts in the airspaces under basal conditions. ${ }^{24}$ Despite this, total leukocyte and neutrophil recruitment into the alveolar spaces were not

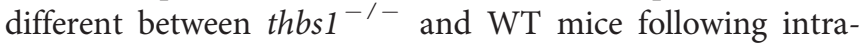
tracheal $K$. pneumoniae (Figure 2a, b). Within the lung parenchymal compartment, thbs $1^{-1-}$ mice showed reduction in IL-6, IL-10, G-CSF, GM-CSF, KC, and MCP-1, but not TNF$\alpha$ concentrations compared with WT controls (Figure 2c). Lung myeloperoxidase (MPO) activity, an indicator of total neutrophil content in tissue homogenates, showed no differences at $48 \mathrm{~h}$ (Figure 2d). By $72 \mathrm{~h}$, however, thbs $1^{-/-}$ lungs showed reduced MPO activity in agreement with reduced total airspace protein concentrations (Figure 2e), a marker of lung injury. Moreover, histologic examination showed lower neutrophil burden in the lungs of $t h b s 1^{-/-}$mice (Figure 2f; Supplementary Figure 2), and semi-quantitative morphometric analysis of lung tissue indicated a significantly lower inflammation score in thbs $1^{-/-}$lungs compared with WT (Figure 2g). These findings suggest that a more effective pulmonary host defense and early containment of bacterial pathogen in thbs $1^{-1-}$ mice are associated with an overall reduction in the intensity of the lung inflammatory response. We next tested the possibility whether the absence of TSP-1 may contribute to enhanced neutrophil function and provide effective containment of bacterial pathogen.

Previous reports have shown the requirement for NE, a serine protease stored within azurophilic granules of neutrophils, for adequate bactericidal activity against Gram-negative bacteria such as Escherichia coli and K. pneumoniae. ${ }^{21,29}$ Lung tissue homogenates of thbs $1^{-1-}$ mice showed enhanced NE compared with the lungs of WT mice at 48 and $72 \mathrm{~h}$, following intratracheal challenge with $K$. pneumoniae (Figure 2h). Taken together, thbs $1^{-1-}$ mice show increased lung NE associated with reduced intensity in the inflammatory response, suggesting the possibility that TSP-1 may regulate NE activity to restrain microbial killing with consequent alterations in the host inflammatory response.

\section{thbs $1^{-1-}$ neutrophils show enhanced microbial killing that is reversed by administration of recombinant TSP-1 or WT serum}

To more closely examine intracellular microbial-killing capacity, thbs $1^{-1-}$ and WT neutrophils were obtained following intraperitoneal inoculation with $K$. pneumoniae. Neutrophils were washed and treated with gentamicin to remove extracellular, membrane-attached bacteria. At time 0 ,

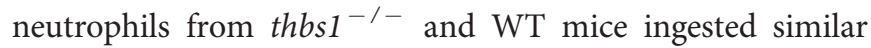
numbers of bacteria as indicated by CFU following lysis of cells (Figure 3a). However, after $60 \mathrm{~min}$ incubation, thbs $1^{-1-}$ neutrophil lysates showed significantly less viable bacteria compared with WT neutrophil lysates (Figure 3a), indicating enhanced microbial killing in the absence of TSP-1. The addition of recombinant TSP-1 just prior to K. pneumoniae challenge in vivo eliminated the enhanced bacterial killing function of thbs $1^{-/-}$neutrophils near to the level of WT neutrophils, suggesting a specific role of TSP-1 in restraining neutrophil microbial killing (Figure 3b). Moreover, whereas WT neutrophils showed a 2.3 -fold reduction in viable bacteria over time with ex vivo incubation, thbs $1^{-/-}$neutrophils showed a 17.8-fold reduction (mean-fold reduction in CFU/10 polymorphonuclear cells \pm s.e.m.: $2.3 \pm 0.2$ vs. $17.8 \pm 6.3$, $P<0.05)$. Compared with thbs $1^{-1-}$ neutrophils treated with vehicle alone, thb1 $1^{-1-}$ neutrophils pretreated with recombinant TSP-1 showed a 5.5-fold reduction over time with ex vivo incubation (mean-fold reduction in CFU/10 polymorphonuclear cells \pm s.e.m.: $17.8 \pm 6.3$ vs. $5.5 \pm 0.3$, $P<0.05)$. Thus, neutrophils show enhanced microbial killing in the absence of TSP-1, and addition of recombinant TSP-1 can mitigate this response.

We next examined microbial killing by WT and thbs $1^{-/-}$ neutrophils following $K$. pneumoniae infection in vitro to determine whether in vitro can recapitulate in vivo findings. At a multiplicity of infection of 50:1, thbs $1^{-/-}$neutrophils showed enhanced microbial killing compared with WT neutrophils (Figure 3c). The enhanced microbial killing observed in thbs $1^{-/-}$neutrophils could be reversed when bacteria incubated with WT serum rather than homologous serum was given to thbs $1^{-/-}$neutrophils (Figure 3d). Moreover, when bacteria incubated in thbs $1^{-/-}$serum was given to WT neutrophils, these neutrophils showed enhanced microbial killing compared with WT neutrophils that received bacteria incubated in homologous serum (Figure 3e). These experiments collectively indicate that neutrophil microbial killing is altered by TSP- 1 and that the major source of TSP- 1 is extracellular in origin.

thbs $1^{-1-}$ neutrophils show enhanced NE and CG activity, but normal respiratory burst and morphology

Previous reports have shown that TSP-1 can bind and competitively inhibit purified CG and NE in vitro. ${ }^{3,4}$ However, it is unknown whether TSP-1 can constrain neutrophil proteolytic function by inhibiting granule serine protease activity. As NE is the predominant serine protease critical in non-oxidative effector killing of microbial pathogen and degrades outer membrane protein $\mathrm{A}(\mathrm{OmpA})$ on the surface of Gram-negative bacteria, ${ }^{18,21,29,30}$ we examined the NE activity of tissue-recruited peritoneal-derived $t h b s 1^{-/-}$and WT neutrophils. Thbs $1^{-/-}$neutrophils showed enhanced NE activity compared with WT neutrophils, as measured by the rate of enzymatic hydrolysis of substrate $N$-methoxysuccinylAla-Ala-Pro-Val p-nitroanilide (Figure 4a). Whereas 
a

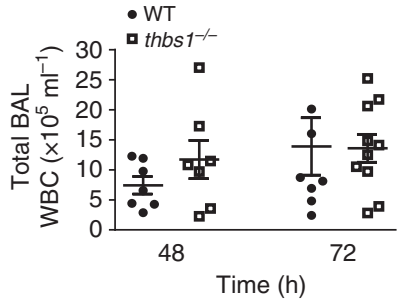

b

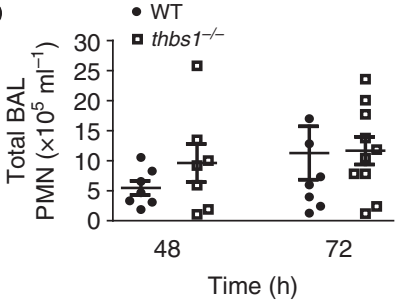

C

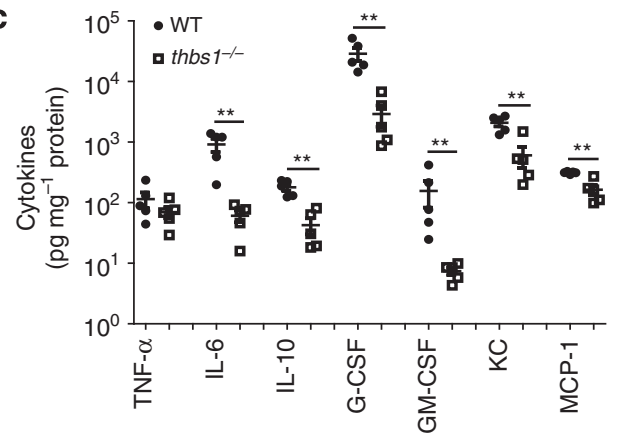

d

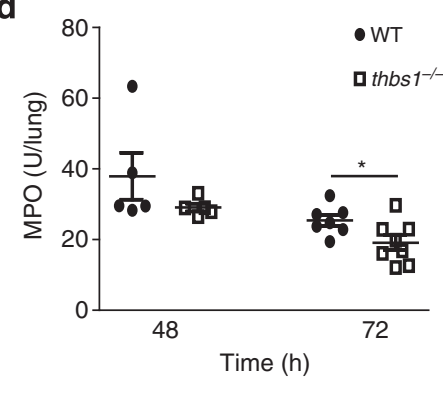

e

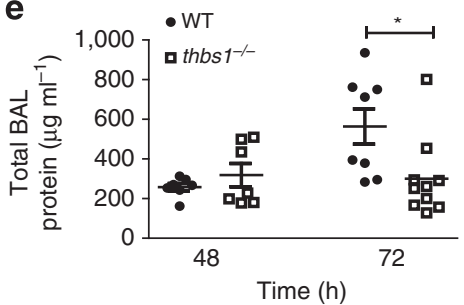

g

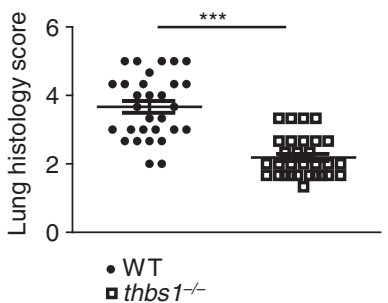

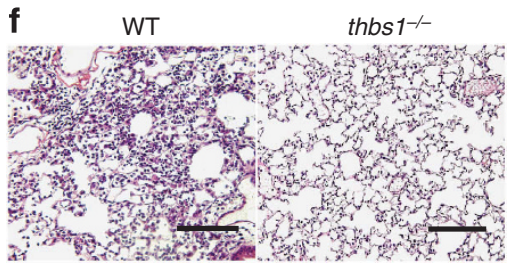

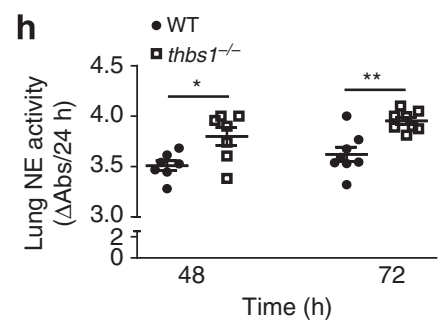

Figure 2 Reduced inflammatory response in the lung parenchyma of thbs $1^{-1-}$ mice is associated with enhanced neutrophil elastase (NE) activity following bacterial pneumonia with $K$. pneumoniae. (a) Total bronchoalveloar lavage (BAL) leukocyte counts and (b) Total BAL polymorphonuclear cell (PMN) counts obtained from wild-type (WT) and thbs $1^{-1-}$ mice 48 and $72 \mathrm{~h}$ following intratracheal (i.t.) inoculation with K. pneumoniae. (c) Lung cytokine measurements in $\mathrm{g} \mathrm{m} \mathrm{m}^{-1}$ from $100 \mu \mathrm{g}$ protein of tissue homogenates obtained from the left lung of mice $48 \mathrm{~h}$ following i.t. K. pneumoniae. Cytokines were measured individually by enzyme-linked immunosorbent assay, and data are presented in one graph for simplicity. (d) Myeloperoxidase (MPO) activity in left lung tissue homogenates measured as U/lung from WT and thbs $1^{-1}$ mice 48 and $72 \mathrm{~h}$ after i.t. inoculation with K. pneumoniae. (e) Total BAL protein concentrations in $\mu \mathrm{g} \mathrm{ml}^{-1}$ from WT and thbs $1^{-1-}$ mice 48 and $72 \mathrm{~h}$ after i.t. inoculation with $K$. pneumoniae. (f) Representative hematoxylin and eosin sections of R lung tissue obtained from WT and thbs $1^{-1-}$ mice at $72 \mathrm{~h}$ post $K$. pneumoniae instillation. Experimental mice underwent BAL of the lung prior to tissue fixation. Scale bar $=100 \mu \mathrm{m}$. (g) Lung histology shows a higher inflammation score in WT compared with thbs $1^{-/-}$mouse lung tissue sections from $72 \mathrm{~h}$ post $K$. pneumoniae instillation, $n=60$ random high-powered images from three sections per group scored by three blinded reviewers. The averaged score for each image is shown as an individual point. (h) NE activity measured in lung tissue homogenates at 48 and $72 \mathrm{~h}$ following i.t. K. pneumoniae. (a-e and h) $n=7-10$ mice per group, Mann-Whitney U-rank sum test, ${ }^{\star \star *} P<0.0001,{ }^{* \star} P<0.01,{ }^{*} P<0.05$. G-CSF, granulocyte-colony stimulating factor; GM-CSF, granulocyte-macrophage colony-stimulating factor; IL, interleukin; KC, keratinocyte-derived chemokine; MCP, monocyte chemoattractant protein-1; TNF- $\alpha$, tumor necrosis factor-alpha; WBC, white blood cells.

$N$-methoxysuccinyl-Ala-Ala-Pro-Val $p$-nitroanilide is an excellent substrate for $\mathrm{NE}$ but not $\mathrm{CG},{ }^{31}$ protease 3 is a neutrophil serine protease contained within azurophilic granules that can also hydrolyze $N$-methoxysuccinylAla-Ala-Pro-Val p-nitroanilide. ${ }^{32-34}$ However, neutrophils obtained from mice deficient in $\mathrm{NE}\left(\mathrm{Ela}^{-{ }^{-}}\right)$ showed minimal to no measureable ability to hydrolyze
$N$-methoxysuccinyl-Ala-Ala-Pro-Val $p$-nitroanilide, indicating that NE accounts for the serine protease activity involved in this reaction (Figure $\mathbf{4 a}$ ).

A mixing experiment was conducted to test whether WT neutrophils possessed a factor that could inhibit the enhanced $\mathrm{NE}$ activity observed in thbs $1^{-/-}$neutrophils. Increasing the ratio of WT neutrophil lysates $(1: 3,2: 3,3: 3)$ added to the 

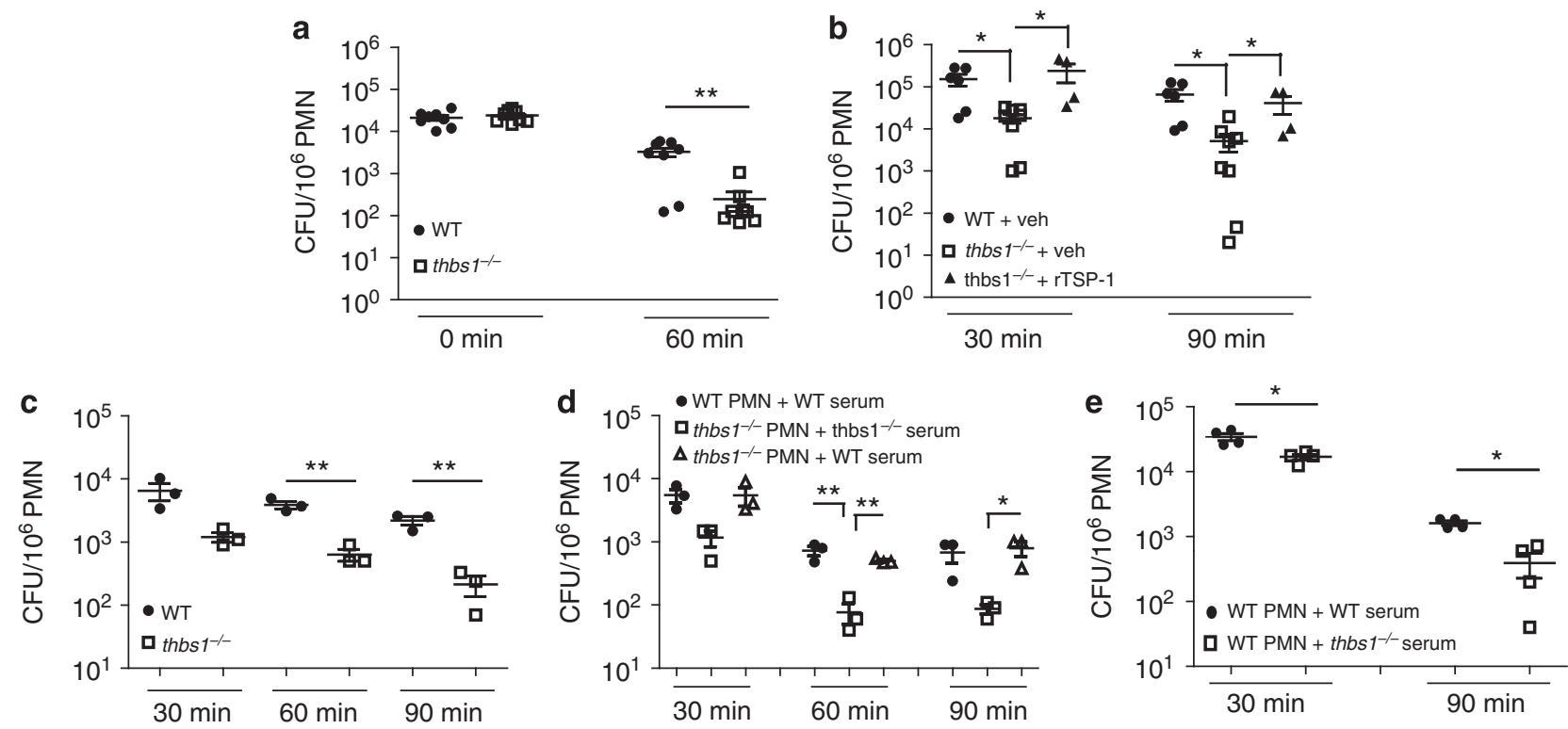

Figure 3 thbs $1^{-1-}$ neutrophils show enhanced intracellular microbial killing that is reversed by administration of recombinant thrombospondin-1 (TSP-1) or wild-type (WT) serum. (a) Intracellular killing by neutrophils from WT and thbs $1^{-1-}$ mice exposed to K. pneumoniae intraperitoneally. Neutrophils were immediately harvested and subsequently plated to quantify colony-forming units (CFUs) or incubated further ex vivo for an additional 60 min prior to plating. CFU $/ 10^{6}$ polymorphonuclear cell (PMN) was obtained for each sample. Data points indicate eight samples per group using harvested neutrophils from four thbs $1^{-1-}$ and four WT mice. (b) Mice were pretreated with recombinant TSP-1 (10 $\mu$ g per mouse) or phosphate-buffered saline vehicle $30 \mathrm{~min}$ prior to intraperitoneal instillation of $K$. pneumoniae. Neutrophils were harvested and subsequently plated to quantify CFU at 30 min or incubated further ex vivo for an additional $60 \mathrm{~min}$ prior to plating. CFU/10 $\mathrm{PMN}$ was obtained for each sample. Data points indicate 4-8 samples per

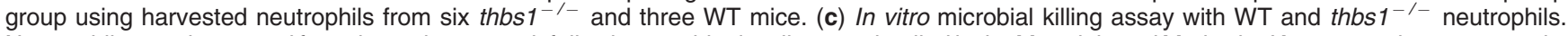
Neutrophils were harvested from the peritoneum $6 \mathrm{~h}$ following $3 \%$ thioglycollate, as detailed in the Materials and Methods. K. pneumoniae was opsonized with $20 \%$ homologous serum on ice for $15 \mathrm{~min}$. Neutrophils at $10^{6}$ per well were infected with K. pneumoniae at a multiplicity of infection of 50 bacteria: 1 neutrophil in vitro. Neutrophils were washed with HBSS + gentamicin to remove extracellular or membrane-attached bacteria, lysed with $0.1 \%$ Triton-X, and CFU determined at the time points indicated. Data points indicate neutrophils harvested from individual mouse, $n=3$ mice per group. (d) In vitro neutrophil microbial killing assay with cross-transfer of WT serum to thbs $1^{-1-}$ neutrophils. Conditions are the same as indicated in c, except thbs $1^{-1-}$ neutrophils received bacteria opsonized with either $20 \%$ homologous or WT serum. Data points indicate neutrophils harvested from individual mouse, $n=3$ mice per group. (e) In vitro neutrophil microbial killing assay with WT neutrophils receiving either bacteria in WT or thbs $1^{-1-}$ serum. Data points indicate neutrophils harvested from individual mouse, $n=4$ mice per group. Mann-Whitney $U$-rank sum test for two group comparisons, Kruskal-Wallis test followed by a Dunn's Multiple Comparisons test for three group comparisons, ${ }^{*} P<0.05,{ }^{* \star} P<0.01$.

substrate mixture containing a fixed amount of thbs $1^{-1-}$ neutrophil lysates dose-dependently reduced the NE activity to the level observed in WT neutrophils (Figure $4 \mathbf{b}$ ). Thus, neutrophils derived from an in vivo environment that lacks TSP-1 show unrestrained NE activity, and reconstitution with lysates from neutrophils derived from mice with intact TSP-1 eliminated this effect. Suc-Ala-Ala-Pro-Phe-pnitroanilide is a chemical substrate for CG but not $\mathrm{NE}^{31}$ and thbs $1^{-/-}$neutrophils also showed enhanced CG activity compared with WT neutrophils (Figure 4c). Taken together, the data indicate TSP-1 regulates both NE and CG activity in neutrophils.

To determine whether TSP-1 alters neutrophil respiratory burst, we measured the oxidation of dihydro-rhodamine to rhodamine in thbs1 $1^{-/-}$and WT neutrophils, a clinically relevant test performed on patient neutrophils suspected of NADPH oxidase dysfunction. thbs $1^{-1-}$ neutrophils showed similar respiratory burst to WT neutrophils (Figure 4d), indicating that reactive oxygen species generation is not exaggerated in thbs $1^{-1-}$ neutrophils that may account for the altered pulmonary host defense. We also compared the ultrastructure of thbs $1^{-1-}$ neutrophils with WT neutrophils, and show similar morphology (Figure 4e). Furthermore, thbs $1^{-1-}$ neutrophils show similar in vitro phagocytosis of fluorescently labeled $E$. coli bioparticles compared with WT neutrophils (Figure 4f). Collectively, these findings suggest that TSP-1 impacts neutrophil proteolytic function by restraining $\mathrm{NE}$ and CG activity, but does not significantly alter the respiratory burst, phagocytosis, or morphology.

\section{Peptides generated from the TSP-1 type-III repeat domain inhibit protease activity of neutrophils}

The type-III repeat domain is a region within TSP-1 that harbors possible inhibitory reactive centers bearing striking similarity to consensus sequences derived from the Kazal and Streptomyces subtilisin inhibitor family members. ${ }^{35,36} \mathrm{We}$ generated peptides corresponding to residues 734-742 (DP-9 peptide) and 793-801 (DV-9 peptide), two stretches of eight amino acids within the type-III repeat domain, bearing reactive-site sequence similarity to select members of the Kazal family of subtilisin inhibitors. Moreover, a peptide corresponding to amino-acid residues $734-742$ previously showed the ability to inhibit activity of purified CG in vitro, whereas a peptide corresponding to residues 793-801 showed 
a
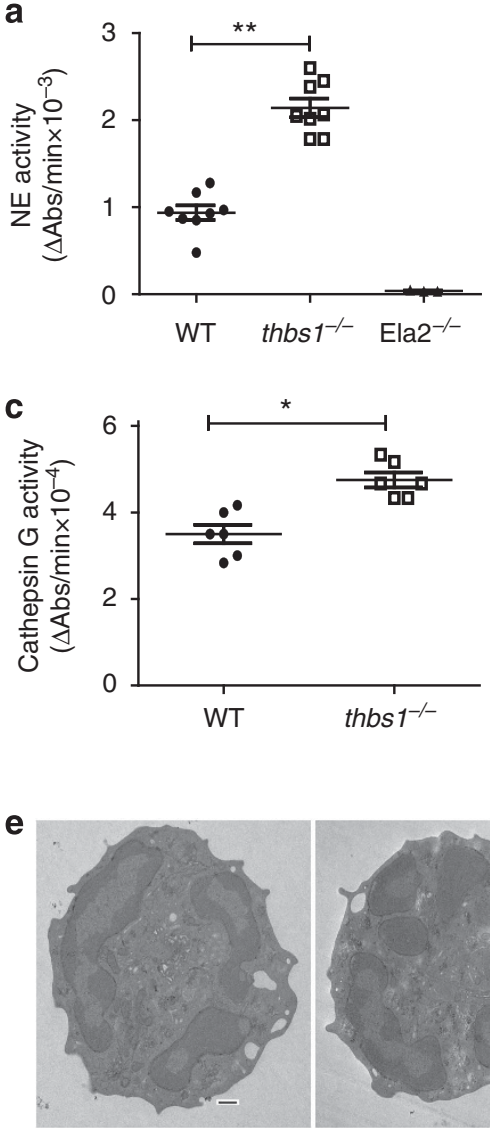

WT
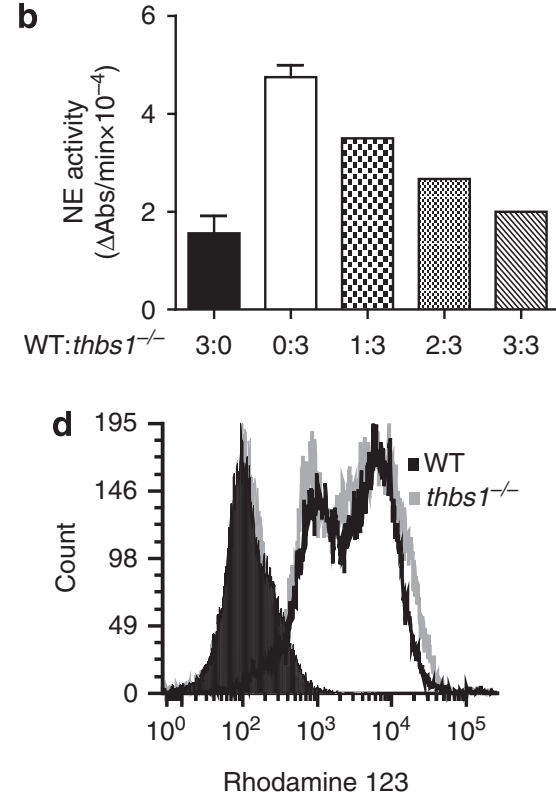

$\mathbf{f}$

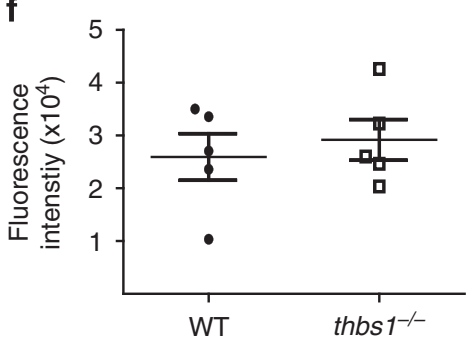

Figure 4 thbs $1^{-1-}$ neutrophils show increased neutrophil elastase (NE) and cathepsin G (CG) activity, but normal neutrophil oxidative burst, in vitro phagocytosis, and morphology. Neutrophils were harvested from the peritoneum $6 \mathrm{~h}$ following $3 \%$ thioglycollate injection. (a) NE activity measured as the rate of enzymatic hydrolysis of synthetic NE substrate $N$-methoxysuccinyl-Ala-Ala-Pro-Val $p$-nitroanilide reflected by the increase in absorbance (Abs) at $405 \mathrm{~nm}$ over time utilizing lysates obtained from wild-type (WT), thbs $1^{-1-}$, and Ela2 ${ }^{-1-}$ neutrophils. Data points indicate neutrophils obtained from four mice per group performed in duplicates. Student's $t$-test, ${ }^{\star \star} P<0.001$. (b) NE activity measured in WT, thbs $1^{-\prime-}$ lysates, and mixtures of WT:thbs $1^{-\prime-}$ neutrophil lysates at differing ratios normalized to protein concentrations. The mixture of neutrophil lysates was generated and pooled from WT and thbs $1^{-1-}$ mice at a ratio of $1: 3,2: 3,3: 3$, where three indicates $30 \mu$ lysate containing $10 \mu \mathrm{g}$ protein. Data obtained from WT (3:0, black bar graph) and thbs $1^{-1-}$ (0:3, white bar graph) neutrophil lysates. (c) CG activity measured as the rate of enzymatic hydrolysis of synthetic CG substrate $N$-succinyl-AlaAla-Pro-Phe- $p$-nitroanilide reflected by the increase in absorbance (Abs) at $405 \mathrm{~nm}$ over time utilizing lysates obtained from WT and thbs $1^{-1}$ neutrophils. Data points indicate neutrophils obtained from three mice per group performed in duplicates. Student's $t$-test, ${ }^{\star} P<0.01$. (d) Respiratory burst of WT and thbs $1^{-1-}$ peritoneal neutrophils as indicated by the shift in fluorescence following oxidation of dihydro-rhodamine $\left(0.4 \mu \mathrm{g} \mathrm{ml}{ }^{-1}\right)$ to rhodamine. Gray-filled histogram: thbs $1^{-/}$neutrophils at baseline; black-filled histogram: WT neutrophils at baseline; gray unfilled histogram: thbs $1^{-1-}$ neutrophils stimulated with phorbol myristate acetate (PMA) $2 \mu \mathrm{g} \mathrm{ml}^{-1}$; black unfilled histogram: WT neutrophils stimulated with PMA $2 \mu \mathrm{g} \mathrm{ml}{ }^{-1}$. Data obtained from neutrophils pooled from five mice per group. (e) Transmission electron microscopy of WT and thbs $1^{-1-}$ neutrophils pooled from five mice per group. Images are representative of 15 neutrophils examined in each group. Scale bars $=500 \mathrm{~nm}$. (f) In vitro phagocytosis of fluorescence-labeled $E$. Coli bioparticles by WT and thbs $1^{-/-}$neutrophils, as indicated by fluorescence intensity. Data points indicate neutrophils obtained from five mice per group performed in quadruplicates.

inhibitory activity to both purified CG and NE. ${ }^{35}$ We generated a third peptide corresponding to residues 854-862 (DA-9) without reactive-site sequence similarity or known inhibitory activity $^{35}$ that was used as control. Unlike in vitro conditions involving purified enzyme and inhibitor of known concentrations, activated neutrophils isolated from thioglycollate-stimulated peritoneum possess numerous proteases and enzymes that can reduce peptide efficacy. Thus, peptides were utilized in excess concentration to determine whether discrete regions of the type-III repeat domain exhibit inhibitory NE and CG activity by a mechanism of substrate competition. Lysates prepared from WT neutrophils were tested for NE activity, as measured by the rate of enzymatic hydrolysis of substrate $\mathrm{N}$-methoxysuccinyl-Ala-Ala-Pro-Val $p$-nitroanilide, in the presence or absence of peptides DV-9, DP-9, and control peptide DA-9.

NE activity was dose-dependently inhibited in the presence of DV-9. DV-9 reduced NE activity by $28 \%$ at $1.25 \mathrm{~mm}$ and $89 \%$ at $2.5 \mathrm{~mm}$ peptide, whereas DP-9 or DA-9 showed no effect (Figure 5a). CG activity, on the other hand, as measured by the rate of enzymatic hydrolysis of substrate $\mathrm{N}$-Suc-Ala-Ala-ProPhe $p$-nitroanilide, was dose-dependently inhibited by DV-9 by $54 \%$ at $1.25 \mathrm{~mm}$ and $86 \%$ at $2.5 \mathrm{~mm}$ (Figure $5 \mathbf{b}$ ). DP-9 at the higher concentration of $2.5 \mathrm{~mm}$ inhibited CG activity by $20 \%$, 

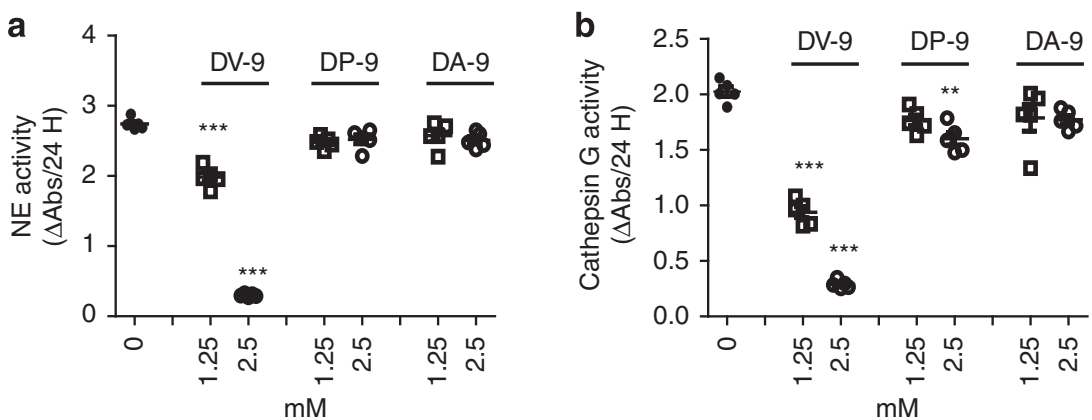

Figure 5 Peptides generated from the thrombospondin-1 type-III repeat domain inhibit neutrophil proteolytic function. (a) Neutrophil elastase (NE) activity measured in wild-type (WT) neutrophil lysates with 1.25 or $2.5 \mathrm{~mm}$ peptides DV-9 (DNCQYVYNV), DP-9 (DNCPFHYNP), and DA-9 (DNCPYVPNA). Data points indicate neutrophils obtained from five mice per group. (b) Cathepsin $\mathrm{G}$ activity measured in WT neutrophil lysates 1.25 or $2.5 \mathrm{~mm}$ peptides DV-9 (DNCQYVYNV), DP-9 (DNCPFHYNP), and DA-9 (DNCPYVPNA). Data points indicate neutrophils obtained from five mice per group. Analysis of variance with Bonferroni multiple comparisons test, ${ }^{\star \star} P<0.01,{ }^{\star \star *} P<0.001$. Abs, absorbance.

whereas DA-9 showed no effect (Figure 5b). Thus, residues 793-801 within the break sequence of the calcium-binding loops, located in the type-III repeat domain of TSP-1, can restrain neutrophil proteolytic function by effectively inhibiting NE and CG enzymatic activity. Residues 734-742 of TSP-1 showed a weak inhibitory effect on CG enzymatic activity within neutrophils.

\section{Peptides corresponding to residues 793-801 of the type-III repeat domain impair neutrophil microbial killing of $K$. pneumoniae}

We next tested whether administration of peptide DV-9, showing inhibitory activity against NE and CG proteolytic function contained within neutrophil lysates, can alter neutrophil microbial killing. Neutrophils harvested from mice administered DV-9 peptide showed significantly higher viable bacteria at 30 and $90 \mathrm{~min}$ post bacterial inoculation than neutrophils from vehicle-treated mice (Figure 6). Neutrophils from vehicle-treated mice showed a 8.3 -fold reduction in viable bacteria with ex vivo incubation over time, whereas neutrophils from peptide DV-9-treated mice showed a 3.5 -fold reduction in viable bacteria over time (mean-fold reduction in CFU $/ 10^{6}$ polymorphonuclear cells \pm s.e.: $8.3 \pm 2.0$ vs. $3.5 \pm 0.6, P<0.05$ ). Thus, the administration of a peptide corresponding to the type-III repeat region of TSP-1 can impair neutrophil microbial-killing machinery against $K$. pneumoniae.

\section{DISCUSSION}

Mice deficient in TSP-1 show enhanced bacterial clearance from the lungs induced by $K$. pneumoniae, reduced splenic dissemination, and increased survival compared with WT controls. Lungs of thbs $1^{-/-}$mice show enhanced NE activity but reduced parenchymal inflammation and injury, suggesting that early containment of bacterial pathogen and microbial killing reduces overall intensity of the inflammatory response following intratracheal challenge with $K$. pneumoniae. In the complete absence of TSP-1, neutrophil serine protease NE and CG activity are intensified as is intracellular microbial killing in a model dependent upon an effective host response to a live bacterial pathogen. We suggest that TSP-1 provides an endogenous



Figure 6 DV-9 peptide impairs neutrophil microbial killing of K. pneumoniae. Intracellular killing by peritoneal neutrophils from wild-type mice treated with peptide DV-9 or vehicle dimethyl sulfoxide just prior to instillation of $K$. pneumoniae in vivo. Thirty min represents the initial time point sampled, allowing for neutrophils to engulf bacteria in vivo. Neutrophils were harvested and subsequently plated to quantify colony-forming units (CFUs) at $30 \mathrm{~min}$ or incubated further ex vivo for an additional 60 min prior to plating. CFU/10 $10^{6}$ polymorphonuclear cell (PMN) was obtained for each sample. Data points indicate neutrophils harvested from individual mouse, $n=4$ mice per group. ${ }^{*} P<0.05$. Mean-fold reduction in $\mathrm{CFU} / 10^{6} \mathrm{PMN} \pm$ s.e.m. over time: vehicle treatment, $8.3+2.0$ compared with DV-9 treatment, 3.5+0.6, Mann-Whitney U-test, ${ }^{\star} P<0.05$.

mechanism to curtail neutrophil proteolytic function during inflammation and identify a region within the break sequence of the calcium-binding loops, located in the type-III repeat domain of TSP-1, that may be involved in this interaction.

We have recently shown that mice deficient in TSP-1 are prone to experimental lipopolysaccharide-induced lung injury, ${ }^{14}$ a well-known non-infectious inducer of inflammation and injury causing vascular leak. However, with a live bacterial pathogen, thbs $1^{-1-}$ mice show more effective containment of bacteria and reduced lung inflammation and injury. Susceptibility to sterile injury but enhanced ability to combat microbial challenge is a phenotype that is consistent with unbridled neutrophil protease activity. Others have shown improved host defense in thbs $1^{-1-}$ mice against live bacterial or fungal challenge, ${ }^{37,38}$ but the mechanisms for these observations were not well understood. thbs $1^{-/-}$mice showed improved survival in a cecal ligation puncture model of sepsis, involving polymicrobial infection in addition to an intraperitoneal 
challenge with live E. coli bacteria. ${ }^{37}$ In an experimental model of systemic candidasis, thbs $1^{-/-}$mice also showed less dissemination of Candida albicans systemically and improved survival. ${ }^{38}$ Although both studies suggested that TSP-1 impairs phagocytic capacity as a potential mechanism for survival differences following pathogen challenge, it remains unclear how TSP-1 would mediate the defect in phagocytosis. In contrast, we have previously reported WT and thbs $1^{-1-}$ alveolar macrophages show similar ability to phagocytize apoptotic neutrophils in vivo, ${ }^{14}$ and herein, show no significant differences between WT and thbs $1^{-1-}$ neutrophils to phagocytize fluorescently labeled E. coli bioparticles in vitro. Similar to our findings, however, thbs $1^{-1-}$ mice showed reduced fungal burden and reduced host inflammatory response in the kidneys in the C. albicans model, ${ }^{38}$ inviting speculation that effective containment of pathogen in thbs $1^{-1-}$ mice also reduced the burden of inflammation following systemic C. albicans challenge. Indeed, enhanced neutrophil intracellular microbial killing in thbs $1^{-/-}$mice due to unopposed serine protease activity could potentially provide an explanation for the findings in the prior studies.

Non-oxygen-dependent neutrophil microbial killing mechanisms are a critical component of host defense. Mice deficient in $\mathrm{NE}\left(\right.$ Ela2 $\left.^{-/-}\right)$show impaired host defense and survival in Gram-negative bacterial sepsis from intraperitoneal K. pneumoniae or E. coli infection, ${ }^{29}$ systemic candidiasis from $C$. albicans, ${ }^{18}$ and pneumonia from Pseudomonas aeruginosa. ${ }^{39}$ Mice deficient in CG $\left(\mathrm{Cts}^{-/-}\right)$show impaired survival following Staphylococcus aureus ${ }^{18}$ and Streptococcus pneumoniae $^{40}$ infection. Thus, it is not surprising that NE and CG doubleknockout mice (Ela2 ${ }^{-/-} \mathrm{Cts}_{\mathrm{G}}^{-/-}$) show increased susceptibility to fungal infection from Aspergillus fumigatus, despite normal neutrophil recruitment and phagocytic activity, ${ }^{30}$ and essentially worse survival than $C t s G^{-/-}$mice from S. pneumoniae pneumonia with greater degree of lung injury from failure to effectively contain pathogen-derived virulence factors. ${ }^{40}$

Surprisingly, mice deficient in SERPINB1 $\left(\operatorname{serpinb} 1^{-/-}\right)$, a serine protease inhibitor of NE, CG, and proteinase 3, fail to clear $P$. aeruginosa pneumonia and show worse survival and inflammation. ${ }^{41}$ Although SERPINB1 is expressed in the cytoplasm of neutrophils, the data would indicate that SERPINB1 mainly functions to protect against the tissue injurious effects of extracellular neutrophil serine proteases and the degradation of host defense molecules such as surfactant protein D. ${ }^{41,42}$ Interestingly, Ela $2^{-/-} \mathrm{Cts}_{\mathrm{G}}{ }^{-/-}$mice are resistant to endotoxic shock, and are protected from vascular leak and lung injury, ${ }^{30}$ supporting the notion that unmitigated release of neutrophil serine proteases into the extracellular space can lead to fulminant tissue injury. Thus, the phenotype of thbs $1^{-1-}$ mice appears to be the reverse of $\mathrm{Ela2}^{-/-} \mathrm{Cts} \mathrm{G}^{-/-}$ mice. Given the TSP-1 effects on neutrophil microbial killing and intracellular protease activity, our data indicate that TSP-1 may be involved in the early regulation and compartmentalization of neutrophil granule serine proteases.

TSP-1 is a matricellular protein with numerous ligands and potential binding partners in vitro. ${ }^{11}$ In kinetic studies examining
TSP-1 inhibition of NE substrate hydrolysis, 1 mol of TSP-1 trimer binds to $2.7 \pm 0.3 \mathrm{~mol}$ of NE with a site-binding constant of $57 \pm 13 \mathrm{~nm}^{4}{ }^{4}$ In assessing TSP-1 inhibition of CG substrate hydrolysis, $1 \mathrm{~mol}$ of TSP-1 trimer binds to $2.9 \pm 0.4 \mathrm{~mol}$ of CG with a site-binding constant of $7.0 \pm 3.5 \mathrm{~nm}^{3}{ }^{3}$ These site-binding constants were obtained in the presence of calcium, as TSP-1 interactions with NE and CG are sensitive to calcium-dependent conformational changes within the type-III repeat domain of TSP-1. ${ }^{3,4}$ In the absence of calcium, the reversible inhibition of NE and CG by TSP-1 is enhanced. ${ }^{3,4}$ Given the complex nature of TSP-1 binding, predictions of physiological interactions remain challenging, and highlight the importance of clarifying the role of TSP-1 in the context of cell-based and in vivo studies. Various reports in vitro suggest TSP-1, either released in solution or adhered to a variety of ligands or binding partners, bind and induce motility of neutrophils, conceivably directing their migration in tissue. ${ }^{25-28,43}$ We found no evidence that TSP-1 significantly contributes to neutrophil recruitment in our model. Although activated rabbit and human neutrophils produce TSP$1,{ }^{6}$ our findings indicate that the major contributor of TSP- 1 is extracellular in origin, likely released in solution during inflammation and adhering to neutrophils to modulate their activity.

In conclusion, we have identified a novel role for TSP-1 in regulating neutrophil function during the innate immune response to $K$. pneumoniae infection. Our findings suggest that TSP-1 can bridle neutrophil proteolytic activity and microbial killing through the inhibitory action of its type-III repeat domain, providing one mechanism by which TSP-1 regulates the innate immune response. Although the host has evolved mechanisms to curtail the extracellular tissue-damaging effects of neutrophil serine proteases, TSP-1 appears to curb neutrophil proteolytic function with unintended consequences for host defense. Some chronic inflammatory lung diseases that demonstrate protease/anti-protease imbalance paradoxically show impaired ability to effectively kill colonizing bacteria, despite large numbers of neutrophils. Free NE activity in the bronchoalveloar lavage fluid of children, with cystic fibrosis early in life, is a strong independent risk factor for the development of bronchiectasis, ${ }^{44}$ and inability to control infection precedes, initiates, and sustains inflammation in cystic fibrosis airways. ${ }^{45}$ Neutrophils from cystic fibrosis patients show increase in TSP-1 gene expression by microarray compared with neutrophils from control healthy subjects. ${ }^{46}$ Moreover, cystic fibrosis patients show increased circulating activated platelets with formation of heterotypic aggregates and surface P-selectin expression, ${ }^{47,48}$ inviting speculation that excessive TSP-1 release in some inflammatory lung diseases may contribute to neutrophil dysfunction and impaired ability to effectively kill colonizing microbes.

\section{METHODS}

Animals. Animal studies were conducted in accordance with the recommendations in the Guide for the Care and Use of Laboratory Animals of the National Institutes of Health. The animal protocol was approved by the Institutional Animal Care and Use Committee at the 
University of Pittsburgh. C57BL/6J (\#000664) and thbs $1^{-/-}$ (B6.129S2-Thbs $1^{\text {tmlHyn } / J, ~ \# 006141) ~ m i c e ~ w e r e ~ o b t a i n e d ~ f r o m ~ t h e ~}$ Jackson Laboratory (Bar Harbor, ME). Thbs $1^{-1-}$ mice were backcrossed to $\mathrm{C} 57 \mathrm{Bl} / 6$ mice nine times and a colony was subsequently established at the University of Pittsburgh. Ela ${ }^{-/-}$(B6.129X1-

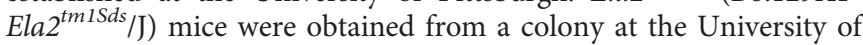
Pittsburgh. All experimental procedures were performed in age(8-12-week old) and gender-matched mice. The animals were housed and maintained in a pathogen-free environment.

Experimental bacterial pneumonia model. K. pneumoniae strain 43816, serotype 2 (American Type Culture Collection, Manassas, VA) was grown in tryptic soy broth overnight at $37^{\circ} \mathrm{C}$. One $\mathrm{ml}$ of this overnight culture was inoculated into fresh tryptic soy broth and grown for $2 \mathrm{~h}$. A standard growth curve of bacterial culture measured by absorbance at $600 \mathrm{~nm}$ was generated to determine mid-log phase of growth. Inoculum concentration, measured in CFU, was determined by serial 10-fold dilutions of bacteria plated on tryptic soy agar plates (Sigma, St Louis, MO). Bacteria was harvested, washed, and resuspended in phosphate-buffered saline before use. WT and thbs $1^{-1-}$ mice were anesthetized with isoflurane, and $10^{3} \mathrm{CFU}$ of $K$. pneumoniae in a total volume of $100 \mu \mathrm{l}$ was carefully administered intratracheally under direct visualization using a sterile $200-\mu \mathrm{l}$ pipet with the filtered tip positioned just above the vocal cords. For mortality studies, $10^{4}$ inoculum was utilized. The inoculums were confirmed by plating serial 10-fold dilutions on tryptic soy broth agar plates.

Bronchoalveloar lavage fluid collection. Animals were euthanized at predetermined time points with isoflurane-inhaled anesthetic overdose within a closed container system, followed by cardiac puncture and exsanguination. Methods for obtaining bronchoalveloar lavage total cell counts and differential and lung histology have been previously reported. $^{24}$

Measurements of lung and spleen bacterial burden. The left lung and spleen were removed following euthanasia at specified time points. For enumerating bacterial CFUs in the lungs and spleens, tissue was homogenized in $1 \mathrm{ml}$ of sterile deionized $\mathrm{H}_{2} 0$. Homogenates $(100 \mu \mathrm{l})$ were plated by 10 -fold serial dilution on tryptic soy agar plates. Bacterial CFU was counted after an overnight incubation at $37^{\circ} \mathrm{C}$.

Measurement of lung cytokines and chemokines. Total protein in lung tissue homogenates were quantified using the Pierce BCA Protein Assay Kit (Thermo Scientific, Rockford, IL). The volume of lung tissue homogenate corresponding to $100 \mu \mathrm{g}$ total protein from each sample was used to perform enzyme-linked immunosorbent assay. Enzymelinked immunosorbent assay duoset antibodies for measuring TNF- $\alpha$, IL-6, IL-10, G-CSF, GM-CSF, KC, MCP-1 were obtained from R\&D Systems (Minneapolis, MN).

MPO assay. Lung tissue homogenates in $0.5 \%$ hexadecyl-trimethylammonium bromide (HTAB) buffer ( $5 \mathrm{~g}$ of hexadecyl-trimethylammonium bromide in 11 of MPO buffer, where MPO buffer contains $6.8 \mathrm{~g}$ of $\mathrm{KH}_{2} \mathrm{PO}_{4}$ and $8.7 \mathrm{~g}$ of $\mathrm{K}_{2} \mathrm{HPO}_{4}$ in $1 \mathrm{l}$ of water) were sonicated for $30 \mathrm{~s}$ and then centrifuged at $20,000 \mathrm{~g}$ for $4 \mathrm{~min}$. Supernatant $(7 \mu \mathrm{l})$ was transferred into a 96-well plate; $200 \mu \mathrm{l}$ of $\mathrm{O}$-dianisidine hydrochloride solution is added immediately prior to reading the optical density at $450 \mathrm{~nm}$ at 0 and $6 \mathrm{sec}$. The MPO activity was calculated using the following formula as previously described: ${ }^{49}$ units of MPO activity in each well $=($ the change in absorbance (between 0 and $60 \mathrm{~s}) /$ time $(\min )) \times 1.13 \times 10^{-2}$.

Neutrophil serine protease activity measurements. NE activity in lung tissue homogenates was measured utilizing a previously described method. ${ }^{50}$ Briefly, $1 \mathrm{mg}$ lung tissue homogenates were incubated with $0.1 \mathrm{moll}^{-1}$ Tris-HCl buffer ( $\mathrm{pH} 8.0$ ) containing $0.5 \mathrm{moll}^{-1} \mathrm{NaCl}$ and $1 \mathrm{mmoll}^{-1} \quad \mathrm{~N}$-methoxysuccinyl-Ala-Ala-Pro-Val $p$-nitroanilide (Sigma) at $37^{\circ} \mathrm{C}$ for $24 \mathrm{~h}$. The degradation of substrate in samples was measured by spectrophotometry at $405 \mathrm{~nm}$. To measure serine protease activity within neutrophils, neutrophils were harvested from the peritoneum at $6 \mathrm{~h}$ following intraperitoneal injection with $2 \mathrm{ml}$ of $3 \%$ thioglycollate. Cell counts were performed manually using a hemocytometer. Cytospins confirmed $>90 \%$ neutrophils at $6 \mathrm{~h}$ harvest. Neutrophils, $1 \times 10^{6}$ per well, were seeded into a 24 -well plate. Non-adherent cells were gently washed off after a 30-min incubation. Remaining adherent cells were lysed with lysis buffer containing leupeptin (Cell Signaling Technology, Danvers, MA). Ten $\mu$ g total protein of lysates from each sample was incubated with $0.1 \mathrm{moll}^{-1}$ Tris- $\mathrm{HCl}$ buffer ( $\mathrm{pH} 8.0$ ) containing $0.5 \mathrm{moll}^{-1} \mathrm{NaCl}$ and $1 \mathrm{mmoll}^{-1}$ of NE substrate $N$-methoxysuccinyl-Ala-Ala-Pro-Val $p$-nitroanilide (Sigma) or CG substrate $N$-Suc-Ala-Ala-Pro-Phe- $p$-nitroanilide at $37^{\circ} \mathrm{C}$ for $1 \mathrm{~h}$, utilizing a modified protocol from Niemann, et al ${ }^{51}$ Peptides DP-9 (Ac-DNCPFHYNP-NH2), DV-9 (Ac-DNCQYVYNVNH2), and DA-9 (Ac-DNCPYVPNA-NH2), corresponding to aminoacid residues 734-742, 793-801, and 854-862 of human TSP-1, respectively, were generated and used in select experiments (Chi Scientific, Maynard, MA). Neutrophil lysates were incubated with $0,1.25$, or $2.5 \mathrm{~mm}$ peptides for $30 \mathrm{~min}$ before NE and CG substrates were added. The reaction was measured by spectrophotometry at $405 \mathrm{~nm}$ over time.

Statistics. Results are reported as the mean plus or minus s.e.m. A Student's two-tailed $t$-test was used to compare two groups and analysis of variance with Bonferroni multiple comparisons test was used for experiments involving $>1$ comparison. For data that was not normally distributed, the Mann-Whitney rank sum test was used to compare two groups and the Kruskal-Wallis test followed by a Dunn's Multiple Comparisons test was conducted for experiments involving $>1$ comparison. Log-rank test was performed to generate the KaplanMeier survival curve. A $P$-value $<0.05$ was considered significant using GraphPad Prism software version 5.0 (La Jolla, CA).

Methods for the transmission electron microscopy, phagocytosis assay, microbial killing assay, and lung histology inflammation scoring are detailed in the online Supplementary Materials section at http:// www.nature.com/mi.

SUPPLEMENTARY MATERIAL is linked to the online version of the paper at http://www.nature.com/mi

\section{ACKNOWLEDGMENTS}

Funding sources include R01 HL086884 (J.S.L.), Flight Attendant Medical Research Institute (J.S.L.), Pilot Project Program in Hemostasis and Vascular Biology through UL1 RR024153 and UL1 TR000005 (University of Pittsburgh CTSI), the Vascular Medicine Institute, the Hemophilia Center of Western Pennsylvania, Institute for Transfusion Medicine (J.S.L.), P01 HL114453 (R.K.M., P.R.), and a Merit Review Award from the US Department of Veterans Affairs (R.K.M.).

\section{DISCLOSURE}

The authors declared no conflict of interest.

c) 2015 Society for Mucosal Immunology

\section{REFERENCES}

1. Frazier, W.A. Thrombospondin: a modular adhesive glycoprotein of platelets and nucleated cells. J. Cell Biol. 105, 625-632 (1987).

2. Bornstein, P. Diversity of function is inherent in matricellular proteins: an appraisal of thrombospondin 1. J. Cell Biol. 130, 503-506 (1995).

3. Hogg, P.J., Owensby, D.A. \& Chesterman, C.N. Thrombospondin 1 is a tight-binding competitive inhibitor of neutrophil cathepsin G. Determination of the kinetic mechanism of inhibition and localization of cathepsin $G$ binding to the thrombospondin 1 type 3 repeats. J. Biol. Chem. 268, 21811-21818 (1993)

4. Hogg, P.J., Owensby, D.A., Mosher, D.F., Misenheimer, T.M. \& Chesterman, C.N. Thrombospondin is a tight-binding competitive inhibitor of neutrophil elastase. J. Biol. Chem. 268, 7139-7146 (1993). 
5. Lawler, J. The structural and functional properties of thrombospondin. Blood 67, 1197-1209 (1986).

6. Kreis, C., La Fleur, M., Menard, C., Paquin, R. \& Beaulieu, A.D. Thrombospondin and fibronectin are synthesized by neutrophils in human inflammatory joint disease and in a rabbit model of in vivo neutrophil activation. J. Immunol. 143, 1961-1968 (1989).

7. Catena, R. et al. Bone marrow-derived Gr1 + cells can generate a metastasis-resistant microenvironment via induced secretion of thrombospondin-1. Cancer Discov. 3, 578-589 (2013).

8. Iruela-Arispe, M.L., Bornstein, P. \& Sage, H. Thrombospondin exerts an antiangiogenic effect on cord formation by endothelial cells in vitro. Proc. Natl. Acad. Sci. USA 88, 5026-5030 (1991).

9. Sweetwyne, M.T. \& Murphy-Ullrich, J.E. Thrombospondin1 in tissue repair and fibrosis: TGF-beta-dependent and independent mechanisms. Matrix Biol. 31, 178-186 (2012).

10. Lawler, P.R. \& Lawler, J. Molecular basis for the regulation of angiogenesis by thrombospondin-1 and -2. Cold Spring Harb. Perspect. Med. 2, a006627 (2012).

11. Resovi, A., Pinessi, D., Chiorino, G. \& Taraboletti, G. Current understanding of the thrombospondin-1 interactome. Matrix Biol. 37, 83-91 (2014).

12. Idell, S. et al. Platelet-specific alpha-granule proteins and thrombospondin in bronchoalveolar lavage in the adult respiratory distress syndrome. Chest 96, 1125-1132 (1989).

13. Lawler, J. et al. Thrombospondin-1 is required for normal murine pulmonary homeostasis and its absence causes pneumonia. J. Clin. Invest. 101, 982-992 (1998).

14. Zhao, Y. et al. Thrombospondin-1 triggers macrophage IL-10 production and promotes resolution of experimental lung injury. Mucosal Immunol. 7, 440-448 (2013).

15. Poe, S.L. et al. STAT1-regulated lung MDSC-like cells produce IL-10 and efferocytose apoptotic neutrophils with relevance in resolution of bacterial pneumonia. Mucosal Immunol. 6, 189-199 (2013).

16. Ranieri, V.M. et al. Acute respiratory distress syndrome: the Berlin definition. JAMA 307, 2526-2533 (2012).

17. Klebanoff, S.J. lodination of bacteria: a bactericidal mechanism. J. Exp. Med. 126, 1063-1078 (1967).

18. Reeves, E.P. et al. Killing activity of neutrophils is mediated through activation of proteases by K+ flux. Nature 416, 291-297 (2002).

19. Segal, A.W. How neutrophils kill microbes. Annu. Rev. Immunol. 23, 197-223 (2005).

20. Ledford, J.G., Kovarova, M. \& Koller, B.H. Impaired host defense in mice lacking ONZIN. J. Immunol. 178, 5132-5143 (2007).

21. Belaaouaj, A., Kim, K.S. \& Shapiro, S.D. Degradation of outer membrane protein A in Escherichia coli killing by neutrophil elastase. Science $\mathbf{2 8 9}$, 1185-1188 (2000).

22. Lee, C.T. et al. Elastolytic activity in pulmonary lavage fluid from patients with adult respiratory-distress syndrome. N. Engl. J. Med. 304, 192-196 (1981).

23. Hogg, P.J., Stenflo, J. \& Mosher, D.F. Thrombospondin is a slow tightbinding inhibitor of plasmin. Biochemistry 31, 265-269 (1992).

24. Zhao, Y. et al. Thrombospondin-1 triggers macrophage IL-10 production and promotes resolution of experimental lung injury. Mucosal Immunol. 7 , 440-448 (2014).

25. Suchard, S.J., Burton, M.J., Dixit, V.M. \& Boxer, L.A. Human neutrophil adherence to thrombospondin occurs through a CD11/CD18-independent mechanism. J. Immunol. 146, 3945-3952 (1991).

26. Mansfield, P.J., Boxer, L.A. \& Suchard, S.J. Thrombospondin stimulates motility of human neutrophils. J. Cell Biol. 111, 3077-3086 (1990).

27. Mansfield, P.J. \& Suchard, S.J. Thrombospondin promotes both chemotaxis and haptotaxis in neutrophil-like HL-60 cells. J. Immunol. 150, 1959-1970 (1993).

28. Suchard, S.J., Boxer, L.A. \& Dixit, V.M. Activation of human neutrophils increases thrombospondin receptor expression. J. Immunol. 147, 651-659 (1991).
29. Belaaouaj, A. et al. Mice lacking neutrophil elastase reveal impaired host defense against gram negative bacterial sepsis. Nat. Med. 4, 615-618 (1998).

30. Tkalcevic, J. et al. Impaired immunity and enhanced resistance to endotoxin in the absence of neutrophil elastase and cathepsin G. Immunity 12, 201-210 (2000).

31. Nakajima, K., Powers, J.C., Ashe, B.M. \& Zimmerman, M. Mapping the extended substrate binding site of cathepsin $G$ and human leukocyte elastase. Studies with peptide substrates related to the alpha 1-protease inhibitor reactive site. J. Biol. Chem. 254, 4027-4032 (1979).

32. Korkmaz, B., Horwitz, M.S., Jenne, D.E. \& Gauthier, F. Neutrophil elastase, proteinase 3, and cathepsin $\mathrm{G}$ as therapeutic targets in human diseases. Pharmacol. Rev. 62, 726-759 (2010).

33. Kam, C.M. et al. Substrate and inhibitor studies on proteinase 3. FEBS Lett. 297, 119-123 (1992).

34. Rao, N.V. et al. Characterization of proteinase-3 (PR-3), a neutrophil serine proteinase. Structural and functional properties. J. Biol. Chem. 266, 9540-9548 (1991).

35. Hogg, P.J., Jimenez, B.M. \& Chesterman, C.N. Identification of possible inhibitory reactive centers in thrombospondin 1 that may bind cathepsin $G$ and neutrophil elastase. Biochemistry 33, 6531-6537 (1994).

36. Lu, S.M. et al. Predicting the reactivity of proteins from their sequence alone: Kazal family of protein inhibitors of serine proteinases. Proc. Natl. Acad. Sci. USA 98, 1410-1415 (2001).

37. McMaken, S. et al. Thrombospondin-1 contributes to mortality in murine sepsis through effects on innate immunity. PLoS One 6, e19654 (2011).

38. Martin-Manso, G. et al. Endogenous thrombospondin-1 regulates leukocyte recruitment and activation and accelerates death from systemic candidiasis. PLoS One 7, e48775 (2012).

39. Hirche, T.O. et al. Neutrophil elastase mediates innate host protection against Pseudomonas aeruginosa. J. Immunol. 181, 4945-4954 (2008).

40. Hahn, I. et al. Cathepsin G and neutrophil elastase play critical and nonredundant roles in lung-protective immunity against Streptococcus pneumoniae in mice. Infect. Immun. 79, 4893-4901 (2011).

41. Benarafa, C., Priebe, G.P. \& Remold-O'Donnell, E. The neutrophil serine protease inhibitor serpinb1 preserves lung defense functions in Pseudomonas aeruginosa infection. J. Exp. Med. 204, 1901-1909 (2007).

42. Wright, J.R. Immunoregulatory functions of surfactant proteins. Nat. Rev. Immunol. 5, 58-68 (2005).

43. Majluf-Cruz, A. et al. Residues F16-G33 and A784-N823 within platelet thrombospondin-1 play a major role in binding human neutrophils: evaluation by two novel binding assays. J. Lab. Clin. Med. 136, 292-302 (2000).

44. Sly, P.D. et al. Risk factors for bronchiectasis in children with cystic fibrosis. N. Engl. J. Med. 368, 1963-1970 (2013).

45. Armstrong, D.S. et al. Lower airway inflammation in infants with cystic fibrosis detected by newborn screening. Pediatr. Pulmonol. 40, 500-510 (2005).

46. Adib-Conquy, M. et al. Neutrophils in cystic fibrosis display a distinct gene expression pattern. Mol. Med. 14, 36-44 (2008).

47. O'Sullivan, B.P. \& Michelson, A.D. The inflammatory role of platelets in cystic fibrosis. Am. J. Respir. Crit. Care Med. 173, 483-490 (2006).

48. O'Sullivan, B.P. et al. Platelet activation in cystic fibrosis. Blood 105, 4635-4641 (2005).

49. Jeyaseelan, S., Chu, H.W., Young, S.K. \& Worthen, G.S. Transcriptional profiling of lipopolysaccharide-induced acute lung injury. Infect. Immun. 72, 7247-7256 (2004).

50. Ishii, T. et al. Neutrophil elastase contributes to acute lung injury induced by bilateral nephrectomy. Am. J. Pathol. 177, 1665-1673 (2010).

51. Niemann, C.U. et al. Neutrophil elastase depends on serglycin proteoglycan for localization in granules. Blood 109, 4478-4486 (2007). 